\title{
Species of coagulase-negative staphylococci isolated from catheter tips from open-heart surgery patients
}

\author{
R FREEMAN AND N HJERSING \\ From the Department of Microbiology, Freeman Hospital, Newcastle upon Tyne
}

ABSTRACT Coagulase-negative staphylococci isolated from the tips of catheters used in open-heart surgical patients were classified into species and the results compared with those obtained by speciation of coagulase-negative staphylococci isolated as blood culture contaminants. Staphylococcus epidermidis was the most common species in both populations, but other species occurred more often in the catheter tip group. Staphylococcus saprophyticus was found in $17 \%$ of the catheter tip series and was not found at all in the blood culture contaminants. Species other than $S$ epidermidis, and particularly $S$ saprophyticus, were isolated more often from catheters which had been in situ some days. These findings suggest that speciation of coagulase-negative staphylococci from catheter tips may point to some species being better adapted to colonise and survive on prosthetic materials. In this study such evidence suggested that $S$ saprophyticus might be such a species. It is concluded that if the initial results presented here are confirmed, a more logical approach to the chemoprophylaxis of prosthetic implant surgery would be possible.

The isolation of coagulase-negative staphylococci from the tips of indwelling intravenous and intra-arterial catheters has been described in patients undergoing open-heart surgery. ${ }^{12}$ In those earlier reports it was established that the incidence of the problem could be reduced by early removal of the catheters, and that the problem was common to all indwelling intravascular catheters in these patients, regardless of site or use. It was further pointed out that coagulase-negative staphylococci figure largely in all series of postoperative prosthetic valve endocarditis and that, therefore, this reservoir of similar organisms might be a source of this form of endocarditis.

One of the problems complicating such studies in the past has been the lack of a simple and rapid method of distinguishing strains of coagulase-negative staphylococci from one another. Recently such a method has become available in the form of the API-staph system, which is a rapid and simple way of classifying these organisms after a biochemical scheme devised by Kloos and Schleifer. ${ }^{3}$ In this report we describe the results of applying this method to the coagulase-negative staphylococci recovered from catheter tips.

Addiess for reprint requests: Dr R Freeman, Department of Microbiology, Freeman Hospital, Newcastle upon Tyne.

\section{Methods}

Catheter tips were obtained and cultured as described earlier. Briefly, this began with the removal of the catheter under aseptic conditions, the severing of the tip with sterile scissors, and the collection of the tip into a sterile container. On receipt of the container in the laboratory, broth was added and any organisms recovered were isolated and identified. Staphylococci so isolated were investigated for coagulase production. One hundred coagulase-negative staphylococci from this source were investigated using the API-staph system.

As controls, 50 strains of coagulase-negative staphylococci isolated from blood cultures were also investigated. Because of the possibility of laboratory contamination of this latter source, these strains were restricted to ones recovered on first sampling of the previously unopened blood culture bottle. Similarly, any blood culture isolates from cultures taken via a catheter were excluded from this group, as were strains from blood cultures taken from the heart-lung machine.

It was possible in 59 instances in which coagulase-negative staphylococci were recovered from a catheter tip sent from an open-heart surgical patient to determine accurately the time 
interval between the insertion of the catheter and its subsequent removal. In all these instances the catheters had been inserted in the operating theatre under full aseptic conditions. All the open-heart surgery patients were receiving prophylactic flucloxacillin throughout this time.

API-staph investigations were performed strictly according to the manufacturer's instructions, results being read at 24 hours. The system comprises 20 biochemical tests which are then scored according to the results obtained into a numerical profile. The various profiles are then grouped into collections, each of which corresponds to one of the species designated by Kloos and Schleifer. ${ }^{3}$ The species so designated are: Staphylococcus epidermidis, $S$ hominis, $S$ haemolyticus, $S$ capitis, $S$ warneri, $S$ saprophyticus, and $S$ simulans. Other species exist, for instance $S$ cohnii, and not all strains are able to be completely assigned. When strains were encountered which had a profile not corresponding to the list supplied, the strain was referred to staff at the API laboratories who kindly verified the profile and where possible assigned the strain to one of the species. Only one strain of the 150 reported here proved impossible to classify.

\section{Results}

Table 1 shows the frequency with which the various species of coagulase-negative staphylococci were found in the two sources. $S$ epidermidis was the most common species in both populations, but whereas it accounted for $80 \%$ of the blood culture contaminants, it was found in only $56 \%$ of the catheter tip organisms. The relative prevalences of $S$ hominis, $S$ haemolyticus, $S$ capitis and $S$ warneri were not markedly

Table 1 Comparison of the isolation rates of the various species of coagulase-negative staphylococci from the two sources

\begin{tabular}{llr}
\hline Species & \multicolumn{2}{l}{ Source of isolate } \\
\cline { 2 - 3 } & $\begin{array}{l}\text { Blood culture } \\
\text { contaminants }\end{array}$ & Catheter tips \\
\hline S epidermidis & $40(80 \%)$ & $56(56 \%)$ \\
$S$ hominis & $4(8 \%)$ & $10(10 \%)$ \\
S haemolyticus & $2(4 \%)$ & $3(3 \%)$ \\
$S$ capitis & $2(4 \%)$ & $6(6 \%)$ \\
$S$ warneri & $1(2 \%)$ & $6(6 \%)$ \\
$S$ saprophyticus & - & $13(13 \%)$ \\
S saprophyticus/haemolyticus* & - & $4(4 \%)$ \\
S simulans & - & $2(2 \%)$ \\
Unidentified & $1(2 \%)$ & - \\
Total & 50 & 100
\end{tabular}

* Strains designated $S$ saprophyticus/haemolyticus are currently awaiting final identification. It is probable that they will eventually be designated $S$ saprophyticus.
Table 2 Relationship between the species of coagulase-negative staphylococcus isolated and the time interval between insertion and removal of the catheter

\begin{tabular}{|c|c|c|c|}
\hline \multirow[t]{2}{*}{ Species } & \multirow[t]{2}{*}{ Number } & \multicolumn{2}{|c|}{$\begin{array}{l}\text { Time bet } x \text { een catheter insertion and } \\
\text { removal }\end{array}$} \\
\hline & & Range & Mean time in situ \\
\hline $\begin{array}{l}S \text { epidermidis } \\
S \text { hominis } \\
S \text { haemolyticus } \\
S \text { warneri } \\
S \text { saprophyticus } \\
S \text { simulans }\end{array}$ & $\begin{array}{r}37 \\
6 \\
1 \\
5 \\
9 \\
1\end{array}$ & $\begin{array}{l}1-5 \\
2-11 \\
6 \\
1-9 \\
2-11 \\
2\end{array}$ & $\begin{array}{l}2 \cdot 08 \text { days } \\
4 \cdot 33 \text { days } \\
- \\
5 \cdot 20 \text { days } \\
5 \cdot 88 \text { days } \\
-\end{array}$ \\
\hline Total & 59 & & \\
\hline
\end{tabular}

different in the two populations, but $S$ saprophyticus was found in $13 \%$ of the catheter tip o population and not at all in the blood culture contaminants. The small number of strains $\frac{3}{3}$ designated $S$ saprophyticus/haemolyticus are awaiting final identification, but will probably beassigned to $S$ saprophyticus eventually, making $\underset{\infty}{\infty}$ this discrepancy even larger.

Table 2 contains a summary of the correlations between the species isolated from 59 catheter tips and the time in situ of the catheters con-o cerned. All of the catheters yielding species other®

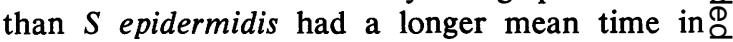
situ than those from which $S$ epidermidis was $\overrightarrow{\vec{\circ}}$ isolated, but this difference was most marked in the subgroup of catheters yielding $S$ saprophyticus.

Table 3 shows the distribution of the isolates of the various species in relation to the day of catheter removal. The rapid decline of the yield of $S$ epidermidis beyond day 3 is seen, in dis- $\dot{0}$ tinction to the continuing isolation of the other species.

Table 3 Distribution by day of the number of isolates of the various species

\begin{tabular}{|c|c|c|c|c|c|c|c|c|c|c|c|}
\hline \multirow[t]{2}{*}{ Species } & \multicolumn{11}{|c|}{ Number of isolates on day } \\
\hline & 1 & 2 & 3 & 4 & 5 & 6 & 7 & 8 & 9 & 10 & 11 \\
\hline$S$ epidermidis & 17 & 7 & 7 & 2 & 2 & - & - & - & - & - & - \\
\hline$S$ hominis & 1 & 2 & 一 & 1 & - & 1 & - & - & - & - & 1 \\
\hline$S$ haemolyticus & - & 一 & - & - & - & 1 & - & - & - & - & 一 \\
\hline$S$ warneri & 1 & 1 & - & - & - & 1 & 一 & 1 & 1 & - & - \\
\hline S saprophyticus & - & 1 & 3 & - & 2 & - & - & 1 & 1 & - & 1 \\
\hline$S$ simulans & - & 1 & - & - & - & - & - & - & - & - & - \\
\hline Total & 19 & 12 & 10 & 3 & 4 & 3 & 0 & 2 & 2 & 0 & 2 \\
\hline
\end{tabular}

\section{Discussion}

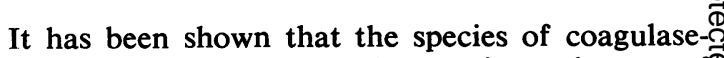
negative staphylococci found in cultures of catheter tips removed from open-heart surgeryo patients differ in their relative frequency in comparison to the distribution of the same specieso 
in a population found as blood culture contaminants, representing skin contamination, in which case the increased frequency of different species in the catheter tip population may be significant. Whereas $S$ epidermidis forms the majority of the isolates in both situations, species other than $S$ epidermidis occur in the catheter tip group, and $S$ saprophyticus is found exclusively in this latter site. Isolation of $S$ epidermidis is associated with catheters removed within three days of their insertion, whereas the other species, and particularly $S$ saprophyticus, are associated with catheters removed after a longer time in situ. $S$ saprophyticus has figured largely in reports of clinical situations in which coagulase-negative staphylococci have been taken to play a pathogenic role, ${ }^{4-6}$ especially in the urinary tract. These findings prompt several further questions.

Firstly, the origin of the strains remains an unresolved question, but several possible explanations can be advanced. Since the population recovered from the catheter tips changes with respect to the species of coagulase-negative staphylococci and with time, it can be argued that skin flora, which the blood culture contaminants would suggest is largely $S$ epidermidis, is introduced early in the lifetime of the catheter, and that some of the minority species (for instance $S$ saprophyticus) come to predominate with the passing of time, either as a result of their being better adapted to survive on the catheter, or as a result of the flucloxacillin. These possibilities might usefully be examined.

Secondly, $S$ saprophyticus might come to lodge on the catheter tip after a subclinical bacteraemia. This might result from catheterisation of the urinary tract since this is universal practice in the immediate postoperative period in open-heart surgical patients.

Thirdly, the possibility that $S$ saprophyticus has a predilection for the material from which catheters are made, or the ability to adhere to such material, has two further implications. It may indicate that some of the strains of coagulase-negative staphylococci isolated from catheter tips are more dangerous than the majority. Thus, an organism with a facility to adhere to plastic materials would be more likely to adhere to the valvular prosthesis itself. The low incidence of postoperative prosthetic valve endocarditis compared to the incidence of isolation of coagulase-negative staphylococci from catheter tips is a discrepancy which might be explicable if only a small subgroup of the staphylococci were likely to settle on the valve. The second point related to this is that such a property in one species of coagulase-negative staphylococci would be of importance in all varieties of prosthetic surgery.

It has long been known that coagulase-negative staphylococci are also responsible for the infective illness which often follows the insertion of Spitz-Holter valves for the relief of hydrocephalus. $^{7}$ In both these instances the link between coagulase-negative staphylococci and prosthetic material is well established. The implication that $S$ saprophyticus is better able to colonise prosthetic material than other staphylococci would be very relevant in these areas of surgery. The relative proportions of the different species of coagulase-negative staphylococci in the above conditions would be valuable information to add to that presented here. Although classification of such isolates has been attempted in the past, 8 the basis of the system used was not that used here. It is interesting to note that an immunecomplex nephritis is found in some proportion of patients with coagulase-negative staphylococcal infection of ventriculo-atrial shunts, ${ }^{9}$ a complication often found in endocarditis. ${ }^{10}$

If it can be shown that the species of coagulase-negative staphylococci found in catheter tip cultures and on other prosthetic material can be subdivided by species and adherence properties, the way is open for a more logical approach to chemoprophylaxis against the most common organism involved in postoperative prosthetic valve endocarditis.

We are grateful to API Laboratories for the provision of API-staph reagents, and to $\mathrm{Dr}$ Thomas of API Laboratories for the provision of a reference service for occasional strains of coagulase-negative staphylococci not identified on initial testing.

\section{References}

1 Freeman R, King B, Hambling MH. Infective complications of open-heart surgery, and the monitoring of infections by the NBT test. Thorax 1973; 28:617-21.

2 Freeman R, King B. Analysis of results of catheter tip cultures in open-heart surgery patients. Thorax 1975; 30:26-30.

3 Kloos WE, Schleifer KH. Simplified scheme for routine identification of human Staphylococcus species. J Clin Microbiol 1975; 1:82-8.

4 Meers PD, Whyte W, Sandys G. Coagulasenegative staphylococci and micrococci in urinary tract infections. J Clin Pathol 1975; 28:270-3.

5 Mortensen N. Studies in urinary tract infections. 
III, Biochemical characteristics of coagulasenegative staphylococci associated with urinary tract infections. Acta Med Scand 1960; 186:47-51.

6 Maskel T. Importance of coagulase-negative staphylococci as pathogens in the urinary tract. Lancet 1974; 1:1155-8.

7 Callaghan RP, Cohen SJ, Stewart GT. Septicaemia due to colonisation of Spitz-Holter valves by staphylococci: five cases treated with methicillin. Br Med J 1961; 1:860-3.
8 Holt R. The classification of staphylococci from $\overline{\overline{\mathrm{G}}}$ colonised ventriculo-atrial shunts. J Clin Pathol 1969; 22:475-82.

9 Stauffer UG. "Shunt nephritis": diffuse glomerulonephritis complicating ventriculo-atrial shunts. Dev Med Child Neurol 1970; 12: supplement 22, $161-4$.

10 Gutman RA, Striker GE, Gilliland BC, Cutler RE. The immune complex glomerulonephritis of bacterial endocarditis. Medicine 1972; 51:1-25. 